\title{
Mechanism of formation of silver nanoparticles in MAG-DMAEMA copolymer aqueous solutions
}

\author{
Dmitry O. Shvedchenko • Tatiana N. Nekrasova • Olga V. Nazarova • \\ Philippe A. Buffat • Elena I. Suvorova
}

Received: 16 March 2015/Accepted: 16 June 2015/Published online: 25 June 2015

(C) Springer Science+Business Media Dordrecht 2015

\begin{abstract}
Dispersed Ag nanoparticles were prepared in aqueous solutions in the presence of pure poly[2(dimethylamino)ethyl methacrylate] (poly-DMAEMA), poly[2-deoxy-2-methacrylamido-D-glucose] (poly-MA $\mathrm{G})$, and their copolymers of poly[MAG-DMAEMA] with different mole fractions. Polymers contributed to the silver reduction, formation of nanoparticles, and stabilization of suspensions. No agglomerations of nanoparticles are formed. For each sample, more than one thousand silver particles were measured by transmission and scanning transmission electron microscopy to determine their number vs diameter and volume versus diameter distributions. The samples with the
\end{abstract}

Electronic supplementary material The online version of this article (doi:10.1007/s11051-015-3083-5) contains supplementary material, which is available to authorized users.

D. O. Shvedchenko · E. I. Suvorova ( $\square)$

A.V. Shubnikov Institute of Crystallography of Russian Academy of Sciences, Leninsky pr., 59, 119333 Moscow, Russia

e-mail: elena.suvorova@epfl.ch; suvorova@ns.crys.ras.ru

T. N. Nekrasova · O. V. Nazarova

Institute of Macromolecular Compounds of Russian

Academy of Sciences, Bolshoy pr., 31,

199004 Saint Petersburg, Russia

P. A. Buffat · E. I. Suvorova

Ecole Polytechnique Fédérale de Lausanne, Centre Interdisciplinaire de Microscopie Electronique,

Station 12, 1015 Lausanne, Switzerland smallest nanoparticle mode diameter of $2.3 \mathrm{~nm}$ were formed in DMAEMA homopolymer suspension, while the mode diameter increased up to $13.3 \mathrm{~nm}$ in copolymers depending on the mole fraction of DMAEMA. A model of Ag nanoparticles' growth taking into account the structure of the copolymers and the amount of reducing centers per monomer is proposed. The volume fraction of large Ag particles ( $>15-20 \mathrm{~nm})$ in the tail of distributions was determined to estimate the part of less efficient nanoparticles assuming that only surface atoms are active. The largest volume occupied by big particles is measured in the solution with pure poly-MAG. Figures of merit, as the ratio of particle area to total volume of particles, were compared for five systems of Ag NPs/polymer. They can be understood from an economical point of view as the total silver investment compared to efficiency.

Keywords Silver nanoparticles - Dispersion of sizes $\cdot$ 2-Deoxy-2-methacrylamido-D-glucose (MAG) · [2-(Dimethylamino)ethyl methacrylate] (DMAEMA) - Activity · Transmission electron microscopy

\section{Introduction}

The main challenge to manufacture silver nanoparticles for applications in catalysis, medicine, and pharmaceuticals is a mass production of stable 
nanoparticles with a narrow size distribution to targetand control-specific effects. It is widely accepted that biological and chemical activities of particles are directly linked to their surface, i.e., for spherical or spheroidal particles; the smaller the diameter, the larger the surface to volume ratio, and the better the particle's biological and chemical activity for a given mass. Green chemistry using polymer-assisted synthesis of Ag nanoparticles and their stabilization is attractive because of biocompatible and ecological technology. Such method lowers the environmental impact by replacing the need of strong and hazardous reductants like sodium borohydride and hydrazine hydrate in particular in the field of biomaterials applications (Vigneshwaran et al. 2006; MedinaRamireza et al. 2009; Chevirona et al. 2014; Sharma et al. 2009).

Among the great number of possible polymers and copolymers, poly[2-(dimethylamino)ethyl methacrylate] (DMAEMA) was reported (Sun et al. 2011; Nekrasova et al. 2013; Wang and Yamauchi 2010; Wang et al. 2012; Li and Yamauchi 2013) to be an effective stabilizing and reducing agent in metal nanoparticles manufacturing, possessing bactericide properties, biocompatibility, and therefore valuable for biomedical purposes (Yasuda 1975; Klomp et al. 1979; Korzhikov et al. 2008). To reduce its cytotoxicity and still further improve its biocompatibility, usually several strategies were pursued such as reduction of the molecular weight or introduction of shielding component in the copolymerization process. Therefore, copolymerization with 2-deoxy-2methacrylamido-D-glucose (MAG), which is nontoxic (Korzhikov et al. 2008; Nazarova et al. 2009), was expected to provide good results in reduction process and preparation of effective antiseptic materials.

The following factors are mainly considered in order to determine the process of reduction in aqueous solutions of polymers: (i) concentration of silver ions, (ii) concentration of polymers or the [monomer units in polymer $] /\left[\mathrm{Ag}^{+}\right]$ratio, (iii) molecular weight of the polymer or length of the molecular chain, (iv) temperature, (v) duration of reaction, (vi) $\mathrm{pH}$, and (vii) solvent (Wu et al. 2010; Voronov et al. 2008; Khan et al. 2012; Wang et al. 2005; Sun et al. 2010; Donati et al. 2009; Tangeysh et al. 2012; Wang and Tseng 2009).

It was found using the experimental data of UV-Vis spectroscopy that higher molecular weight polymers have greater reducing capabilities compared to the ones observed from lighter polymers (Wu et al. 2010). Authors explained this by the longer molecular chains providing more reducing sites for $\mathrm{Ag}^{+}$. However, they could not decide if an increased reducing capability leads to larger size or higher particle concentration.

In the present work, we used poly[DMAEMA], poly[MAG] homopolymers, and DMAEMA-MAG copolymers with various mole fractions to create and stabilize the Ag nanoparticles in aqueous solutions. Copolymers with different structures can provide the different amounts of reducing sites and maintain the size of nanoparticles and their dispersions. We aim to suggest a model of systematic change of the particle sizes depending on structure of copolymers that can allow predicting nanoparticle size dispersion in such a system.

Establishing the relationships between nanoparticles sizes and polymers has both the fundamental scientific and practical interests. The narrow singlemodal nanosize distributions are typically preferred for many applications since on the one side, broad particle size can reduce the expected positive chemical or bioeffects, and on the other side may result in overconsumption of expensive materials.

Usually, particle number (or frequency in distributions) versus diameter relationship is proposed for evaluation of the different preparation methods, biological/chemical activities of nanoparticles, and the total economic effects. However, considering volume (or mass) distribution of particles to our opinion is a more actual way to estimate the efficiency of reagents, synthesis route, and conditions in order to allow the way to minimize the fraction of large ineffective Ag particles in the final product. Since large particles have a low surface/volume ratio compared to the small ones, in other words a poor efficiency compared to silver cost, hence, development efforts should aim to reduce the large particle tails in size distributions than to lowering the average size in distribution. To our knowledge, such approach was not developed before.

For this goal, the volume distribution of particles was investigated by transmission, high-resolution transmission, and scanning transmission electron microscopy (TEM-HRTEM-STEM) to estimate which part of the total amount of silver contains the smallest, useful particles. Imaging by transmission electron microscopy (TEM) and sizing the particles allowed us to obtain their diameter probability density 
function and therefore calculate the volume fraction of particles for a given diameter. One should mention that, though often used, X-ray diffraction (XRD) using the width of broaden peaks to deduce the particle size according to the Scherrer equation would have been simply wrong because it is only valid for randomly orientated particles of unique size and shape (Guinier 1994), which is obviously not the case here. For instance, the size distribution shape may easily introduce a shift in the apparent average particle size up to $50 \%$ for lognormal distributions as it can be deduced from Rao and Houska (1986) or even more for bimodal distributions and distributions with a long tail. Therefore, TEM is the only accurate method able to cover a broad range of sizes extending from $100 \mathrm{~nm}$ down to the nanometer though it is more time consuming.

Figures of merit, defined as the ratio of the cumulative distribution of the particle surface area to the total mass of silver, are reported for five samples of Ag NPs/polymer. These data might provide a novel approach and help choose the best path for the manufacturing of the Ag nanoparticles for specific bioapplications.

\section{Materials and methods}

All initial reagents for polymerization and Ag nanoparticles preparation are as follows: 2-(dimethyl amino)ethyl methacrylate (DMAEMA), (+)—Dglucosamine hydrochloride, methacryloyl chloride, triethylamine, 2,2'-azobisisobutyronitrile (AIBN), dimethylformamide (DMFA), and $\mathrm{AgNO}_{3}$ were purchased from Aldrich (Germany). Before use, triethylamine, DMAEMA, methacryloyl chloride, and DMFA were distilled under ambient conditions.

The synthesis of MAG was performed according to the process of Korzhikov et al. (2008) using (+) -Dglucosamine hydrochloride, triethylamine, and methacryloyl chloride amine at $-5^{\circ}$ and afterward brought to room temperature during ca. $6 \mathrm{~h}$. The product was filtered and washed with chloroform, dried under vacuum, and finally recrystallized in ethanol.

\section{Polymerization}

The copolymers were obtained by radical copolymerization under argon in sealed ampoules at $60{ }^{\circ} \mathrm{C}$ during $24 \mathrm{~h}$ of a solution of MAG and DMAEMA in dimethylformamide in the presence of AIBN as an initiator (Nazarova et al. 2009). Copolymers were dialyzed through a 1000-molecular-weight cutoff dialysis tubing (Spectra/Por 7, USA) against $2 \%$ $\mathrm{NaCl}$ water solution for $24 \mathrm{~h}$ and then against water for $24 \mathrm{~h}$, and afterward, copolymers were lyophilized. The composition of MAG/DMAEMA copolymers was determined by ${ }^{1} \mathrm{H}$ NMR spectroscopy (Bruker Avance 400 spectrometer, Germany; D2O solution) and potentiometric titration of DMAEMA units with $\mathrm{HCl}$ solution (pH-410 pH-meter, Akvilon, Russia). Both methods gave the same estimation of DMAEMA in the corresponding copolymers.

Synthesis of the Ag nanoparticles

Silver nanoparticles were synthesized by the chemical reduction of $0.3 \mathrm{M}$ silver nitrate in aqueous solutions of $2 \mathrm{wt} \%$ MAG/DMAEMA copolymers or pure DMAEMA and MAG homopolymers at room temperature and pressure. The homopolymer/copolymer concentrations were varied from 10 to $30 \mathrm{mg} / \mathrm{mL}$, the [monomer]: $\left[\mathrm{AgNO}_{3}\right]$ molar ratio was $7: 9$, and $\mathrm{pH}$ was kept in the range of 7.0-7.5. More synthesis details are given in (Nekrasova et al. 2013).

The final Ag NP suspensions were kept at room temperature during $24 \mathrm{~h}$ and then dialyzed against water for $24 \mathrm{~h}$ and lyophilized. The dry samples could be again dissolved in water for use.

UV-Vis monitoring

The progress of the chemical process was monitored by following UV-Vis absorbance spectra of the silver nanoparticles/polymer suspensions with a Specord M40 spectrometer (Carl Zeiss, JENA). Spectra exhibited plasmon bands in the range of 405-420 nm which corresponds to the presence of $\mathrm{Ag}$ nanoparticles. The absence of $\mathrm{Ag}^{+}$ions was controlled using the reaction with $\mathrm{NaCl}$ solutions.

\section{TEM-HRTEM-STEM investigation}

Samples of Ag nanoparticle-polymer water suspensions (1.0-2.0- $\mu$ l droplets) lying on carbon films $/ \mathrm{Cu}$ grids after drying were investigated by transmission (TEM), high-resolution transmission (HRTEM) electron microscopy, and scanning transmission electron microscopy (STEM) in a FEI Tecnai Osiris 
microscope (200 kV X-FEG field-emission gun, first condenser aperture diameter $2000 \mu \mathrm{m}$, second condenser aperture $70 \mu \mathrm{m}$, objective aperture 10-20 $\mu \mathrm{m}$, $4 \mathrm{k} \times 2.6 \mathrm{k}$ pixels Gatan Orius CCD camera and FEI Smart CAM CCD camera instead of conventional fluorescent screen).

\section{Results and discussion}

Duration of reactions and latent periods

Homopolymers DMAEMA, MAG, and copolymers MAG-DMAEMA progressively reduce the silver ions to form Ag nanoparticles. Latent periods of $12 \mathrm{~h}$ for pure poly-MAG and $1 \mathrm{~h}$ for pure poly-DMAEMA were observed before the silver plasmon band appeared in UV-Vis spectra indicating that the formation of Ag NPs had started. The progress of the redox process was followed until the complete disappearance of $\mathrm{Ag}^{+}$ions in the solution was observed for pure poly-MAG (24 h) and poly-DMAEMA (12 h). In contrast, MAG-DMAEMA copolymers (Table 1) do not exhibit any latent periods and the full reaction duration was about $3 \mathrm{~h}$.

The absence of latent period and relatively short duration of reaction to complete the reduction of silver ions makes copolymers very attractive in different applications.

We attribute the shortening of the latent periods up to zero and the decrease of the duration of reactions for copolymers to the stronger intramolecular interactions in their chains compared to the homopolymers. The relaxation times determined by Andreeva et al. (2014) (Table SM-1 in Supplementary Materials) characterize the nanosecond intramolecular mobility of polymer chains and show that the mobility of MAG chain fragments is higher than the mobility of DMAEMA fragments. Hydrophobic interactions between $\mathrm{CH}_{3}$ groups govern the strong rigidity of poly[DMAEMA] chains. The incorporation of DMAEMA units into copolymers results in the formation of more rigid and compact chain configuration with higher local density of units and optimized spatial distribution of reducing groups in comparison with homopolymers.

\section{Particle/volume distributions derived from TEM/ STEM}

TEM-HRTEM-STEM images show that silver nanoparticles are well-dispersed, single-crystalline spheroids. The larger particles (more than ca. $15 \mathrm{~nm}$ ) take often a more ellipsoid-like shape while still single crystalline. Several crystals are twinned or even multiple twinned (Figure SM-1 in Supplementary Materials).

Size distributions gather the diameters of about 1000 nanoparticles for each sample. Particle diameters were estimated on bright-field TEM or STEM images. Standard methods for automatic size measurement based on gray level thresholding-possibly with some filtering or averaging of noise-fail to precisely size the particles of a very few nanometers diameter where contrast is similar to the grainy structure of the supporting carbon film. Therefore, their size was manually estimated by fitting the area of a circular ring mask to that of the projection of spheroidal particles. In spite of time-consuming procedure and possible subjective choices, we concluded for sample series with particles $<5 \mathrm{~nm}$ in diameter such manual measurements in Digital Micrograph or Image J bring — at present at least-more reliable results than using the automatic particle analysis suggested by the two programs above or some other algorithms. Comparison of size distributions measured on the same micrographs by three analysts show that the effect of the personal subjective estimation of the particle projection edge does not significantly change the result.

Peak (or mode) and average diameters, and standard deviations were determined as well as the distributions of particles area and volume that are

Table 1 Latent periods and duration of reaction

\begin{tabular}{llrr}
\hline Polymer & DMAEMA molar fraction $\alpha(\%)$ & Latent period (h) & Duration of reaction (h) \\
\hline Homopolymer MAG & 0 & 12 & 24 \\
Homopolymer DMAEMA & 100 & 1 & 12 \\
Copolymer MAG-DMAEMA & Any ratio studied here & 0 & 3 \\
\hline
\end{tabular}


relevant for chemical activity and economic considerations.

The smallest Ag NPs with a mode diameter of $2.3 \mathrm{~nm}$ and a very narrow standard deviation of $0.9 \mathrm{~nm}$ were obtained in DMAEMA homopolymer solution (Fig. 1a). All particles observed in this sample are $<10 \mathrm{~nm}$ in diameter, and the corresponding histogram does not have a tail at coarse sizes. This result can be ascribed to the conformational status of copolymer molecules in neutral solutions. Zhou et al. (2009) showed that compact conformation of polyDMAEMA brushes due to strong polymer chainchain interactions may hinder or even prevent diffusion of atoms or clusters particles. In opposite, quite broad size distribution with a large amount of big particles (more than $20 \mathrm{~nm}$ in diameter) and therefore containing a large part of silver mass was obtained in poly[MAG] solution (Fig. 1b).

Copolymerization of MAG and DMAEMA with several molar ratios between the monomers leads to a general increase of the sizes of Ag NPs as well as the standard deviation and an appearance of the tails in the size distributions (Fig. 2a-d). The largest average particle diameter was observed for the copolymer MAG-DMAEMA with the molar ratio of $\alpha=47 \%$ (Fig. 2b). In this case, the mode diameter increased by a factor of 4 compared to the smallest one observed for poly[DMAEMA] sample.

The volume fractions of large particles with diameters located at the tails of distributions are increasing
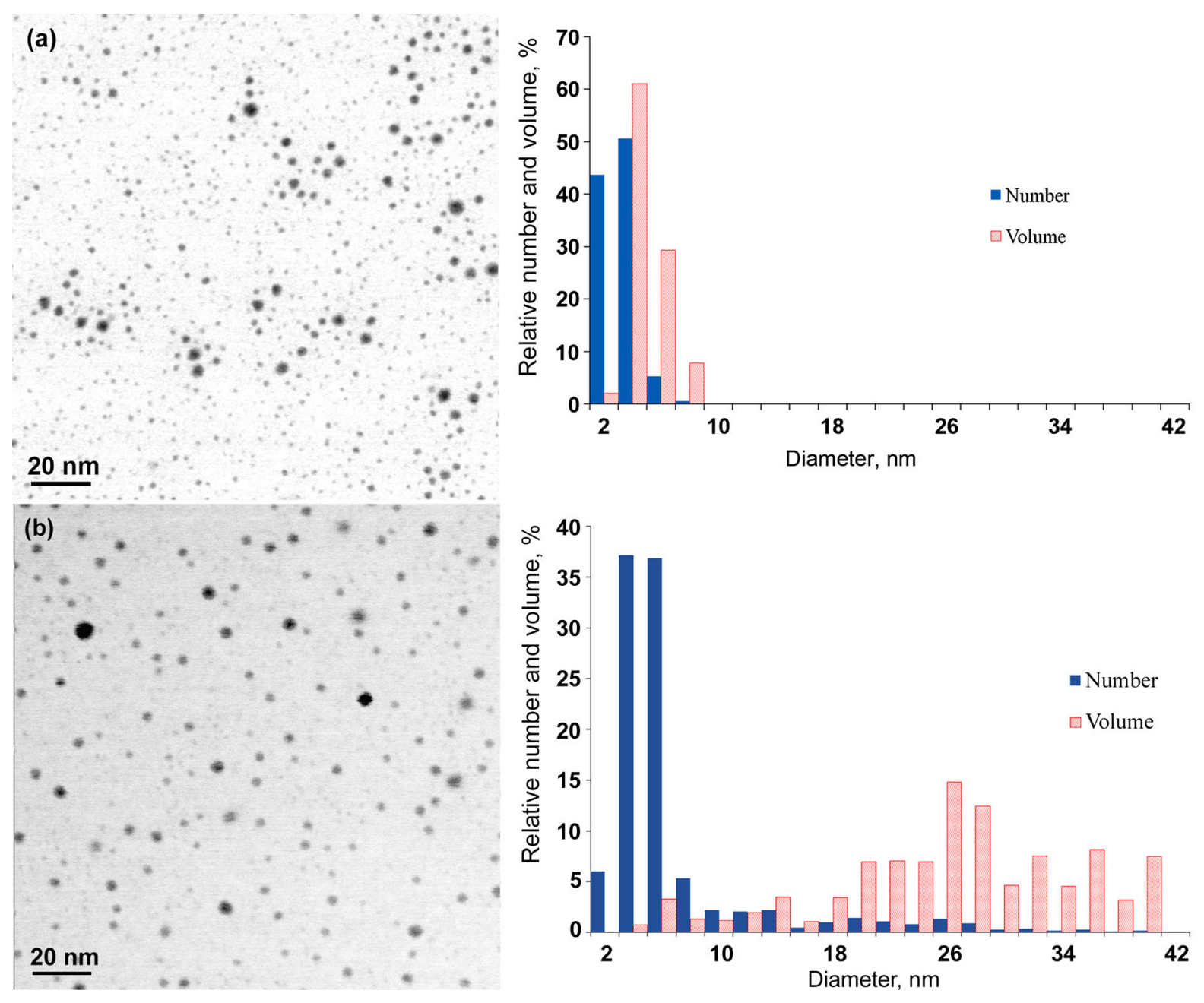

Fig. 1 Bright-field STEM image of AgNPs synthesized with DMAEMA and particle/volume diameter distribution (a), bright-field STEM image of AgNPs synthesized with MAG and particle/volume diameter distribution (b) 

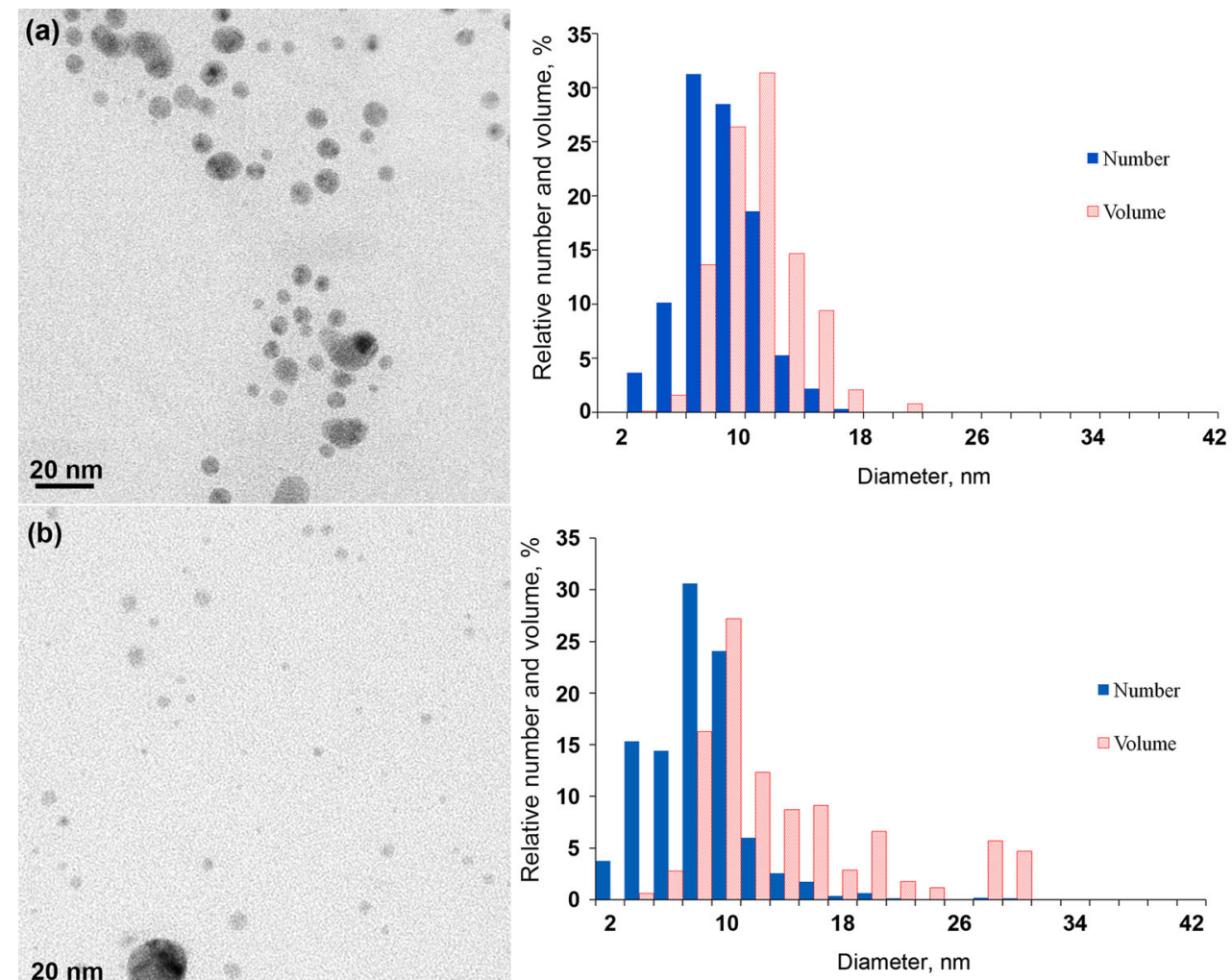

$20 \mathrm{~nm}$
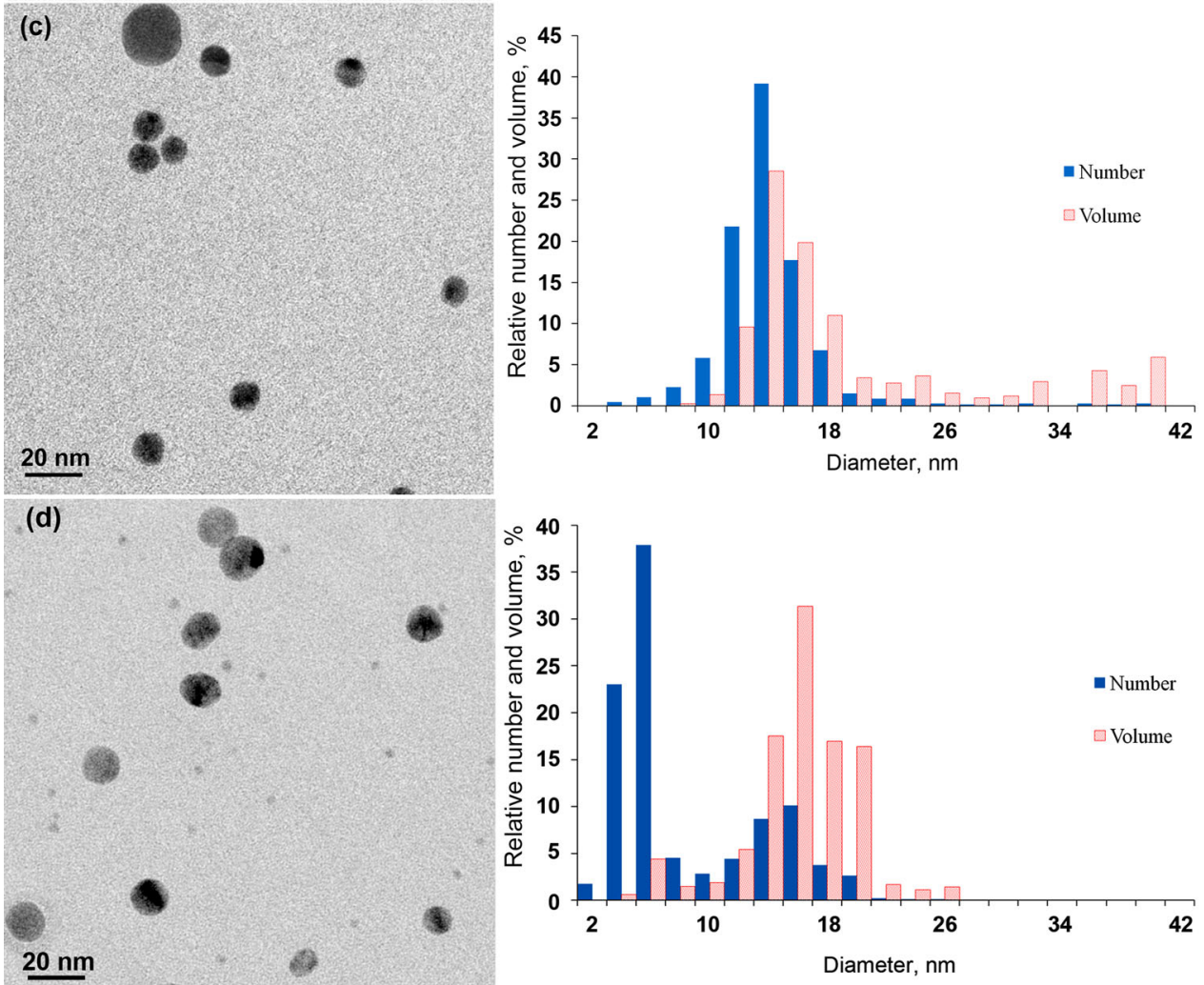
4Fig. 2 TEM micrographs of AgNP synthesized in the MAGDMAEMA copolymer solution with different DMAEMA molar ratio $\alpha$ and the corresponding particle/volume diameter distributions. (a) $\alpha=75.0 \%$, (b) $\alpha=70.7 \%$, (c) $\alpha=47.0 \%$, (d) $\alpha=18.0 \%$

with a change of DMAEMA mole fraction $\alpha$ down to $70.7 \%$ in copolymers (Fig. 2b-d) reaching the maximum $33 \%$ in MAG-70.7 DMAEMA copolymer and finally $94 \%$ in pure poly[MAG]. Further reduction of a volume fraction $\alpha=18.0 \%$ leads to bimodal size distribution with a relatively small tail after the second peak at large diameters in the histogram. The corresponding results are summarized in Table 2 .

Only the MAG-DMAEMA with the 82:18\% ratio samples exhibit bimodal particle distributions. Maxima around 5 and $15 \mathrm{~nm}$ for the copolymer are very distinct on the TEM images (Fig. 2d). The broad single-peaked size distribution is found for Ag NPs formed in pure poly[MAG] solution with small mode diameter of $4 \mathrm{~nm}$. However, this sample gave the bimodal volume distribution and the largest part (up to $94 \%$ of the total volume) of coarse particles among all samples investigated in this work.

\section{Mechanism of formation of Ag nanoparticles}

In order to explain the change in particle mode diameters depending on the polymer structure, we consider that MAG-DMAEMA copolymers are able to provide a suitable environment for reduction of silver ion through the presence of functional carbonyl and amine groups and creation of the local supersaturation of $\mathrm{Ag}$ atoms and constitute a favorable configuration of polymer chains.

Our results show that the Ag particle mode diameter is increasing with the increase of the MAG monomer amount in copolymers (Tables 2, 3). Evaluation of length of polymer molecules in function of monomers concentrations, their molecular weights, and volumes indicates that these parameters have similar order of magnitudes for all samples (Table 3). Therefore, we conclude that MAG and DMAEMA monomers have unequal reducing capabilities and possess the different number of reducing sites.

Each DMAEMA monomer with tertiary amine group provides only one reducing site $i_{\text {DMAEMA }}=1$, while the MAG monomer possesses several potential centers for reduction (Fig. 3). The first one is the carbonyl group linked to ring oxygen (Darroudi et al. 2010). Second, alcohol OH groups in glucose residue can also be oxidized to carboxylic or/and ketone (Vassilyev et al. 1985a, b, Part I and Part II). Third, the oxidative cleavage of the $\mathrm{C}-\mathrm{C}$ bond in the ring can occur with the formation of aldehyde-bearing copolymers (Korzhikov et al. 2008). All together, MAG monomer is able to provide up to $i_{\mathrm{MAG}}=4$ potentially reducing sites (Fig. 3).

We suggest a model which explains the dependence of Ag particle mode diameter with MAG-DMAEMA molar ratio in the copolymer molecule based on proportionality of the particle mode diameter to the average number of reducing sites per monomer.

The average number of reducing sites per monomer in copolymer can be written as $\langle i\rangle=$

Table 2 Diameter (mode and average) of $\mathrm{Ag}$ particles and volume fractions

\begin{tabular}{|c|c|c|c|c|c|c|}
\hline Polymer & $\begin{array}{l}\text { DMAEMA } \\
\text { molar ratio } \alpha \\
(\%)\end{array}$ & $\begin{array}{l}\text { Mode } \\
\text { diameter } \\
(\mathrm{nm})\end{array}$ & $\begin{array}{l}\text { Average } \\
\text { particle } \\
\text { diameter }(\mathrm{nm})\end{array}$ & $\begin{array}{l}\text { Standard } \\
\text { deviation (nm) }\end{array}$ & $\begin{array}{l}\text { Volume fraction } \\
\text { of NP diameter } \\
d \leq 10 \mathrm{~nm}(\%)\end{array}$ & $\begin{array}{l}\text { Volume fraction } \\
\text { of Ag NPs from tail } \\
\text { of distribution }(\%)^{\mathrm{a}}\end{array}$ \\
\hline DMAEMA & 100.0 & 3.4 & 2.34 & 0.89 & 100 & 0 \\
\hline MAG & 0 & 4.0 & 5.99 & 5.81 & 6 & 94 \\
\hline MAG-DMAEMA & 75.0 & 8.4 & 8.62 & 2.48 & 42 & 1 \\
\hline MAG-DMAEMA & 70.7 & 8.4 & 7.04 & 3.33 & 48 & 33 \\
\hline MAG-DMAEMA & 47.0 & 13.4 & 13.27 & 3.68 & 2 & 29 \\
\hline \multicolumn{7}{|l|}{ Bimodal distribution } \\
\hline MAG-DMAEMA & 18.0 & 5 and 15 & 7.57 & 4.88 & 8 & $4^{\mathrm{b}}$ \\
\hline
\end{tabular}

\footnotetext{
${ }^{a}$ Tail of distribution at coarse sizes

b $4 \%$-volume fraction of Ag NP from the tail after the second peak in the bimodal size distribution
} 
Table 3 Molecular mass (MM), polymer molecule length, and number of polymer molecules required for the given mode diameter of the nanoparticle and assumed numbers of reducing sites in MAG monomers

\begin{tabular}{|c|c|c|c|c|c|c|c|}
\hline \multirow[t]{2}{*}{$\alpha(\%)$} & \multirow[t]{2}{*}{$\begin{array}{l}\mathrm{MM} \\
(\mathrm{kDa})\end{array}$} & \multirow{2}{*}{$\begin{array}{l}\text { Mode } \\
\text { diameter, } \\
d(\mathrm{~nm})\end{array}$} & \multirow{2}{*}{$\begin{array}{l}\text { Polymer } \\
\text { molecule } \\
\text { length }(\mathrm{nm})\end{array}$} & \multicolumn{4}{|c|}{$\begin{array}{l}\text { The number of polymer molecules for the corresponding mode diameter and } \\
\text { assumed reducing sites number }\end{array}$} \\
\hline & & & & $\begin{array}{l}1 \text { Reducing } \\
\text { site }\end{array}$ & $\begin{array}{l}2 \text { Reducing } \\
\text { sites }\end{array}$ & $\begin{array}{l}3 \text { Reducing } \\
\text { sites }\end{array}$ & $\begin{array}{l}4 \text { Reducing } \\
\text { sites }\end{array}$ \\
\hline 100.0 & 22 & 3.4 & 21 & 8 & 8 & 8 & 8 \\
\hline 75.2 & 46 & 8.4 & 38 & 65 & 52 & 44 & 37 \\
\hline 70.7 & 37 & 8.4 & 30 & 83 & 64 & 52 & 44 \\
\hline 47.0 & 19 & 13.4 & 14 & 739 & 483 & 359 & 285 \\
\hline 18.0 & 77 & 15.0 & 50 & 286 & 157 & 108 & 83 \\
\hline 0 & 73 & 4.0 & 44 & 6 & 3 & 2 & 2 \\
\hline
\end{tabular}

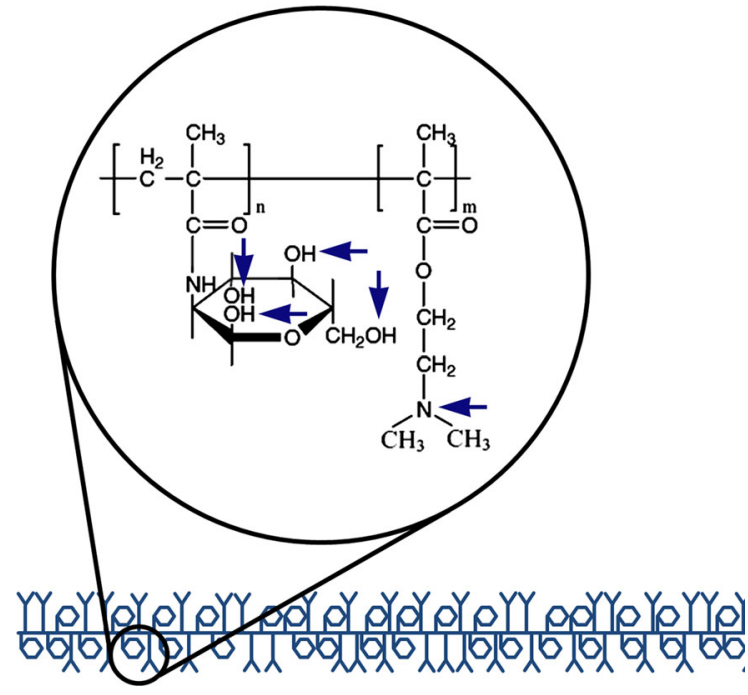

Fig. 3 Structural formula of MAG-DMAEMA copolymer with potentially reducing sites (pointed out by arrows)

$1 \alpha+4(1-\alpha)=4-3 \alpha$, where $\alpha$ is the mole fraction of DMAEMA in copolymers.

In this case, the mode diameter

$d_{\text {mode }} \propto\langle i\rangle=(4-3 \alpha)$.

In order to find the proportionality factor between $d_{\text {mode }}$ and $\langle i\rangle$, we suggest that stabilization of a particle is carried out by the polymer molecules which contributed to reduction of the corresponding silver ions, that is, a silver particle with the mode diameter needs $N_{\mathrm{Ag}}$ of $\mathrm{Ag}$ atoms which were created by means of $\langle i\rangle$ reducing sites from $N_{\mathrm{mm}}$ monomers:

$N_{\mathrm{Ag}}=\langle i\rangle \cdot N_{\mathrm{mm}}$.
For the given Ag particle diameter $d$ (unit cell of Ag contains 4 atoms and the lattice parameter is $0.42 \mathrm{~nm}$ ),

$N_{\mathrm{Ag}}=4 \frac{\pi}{6}\left(\frac{d}{0.42 \mathrm{~nm}}\right)^{3}$

We consider that polymer molecules cover the particle, and the distance between coil turns is comparable with the $\mathrm{C}-\mathrm{C}$ bond length $(\mathrm{C}-\mathrm{C}$ bond length is about $0.15 \mathrm{~nm}$ ). The number of monomers $N_{\mathrm{mm}}$ on the particle surface is estimated as

$N_{\mathrm{mm}}=\pi\left(\frac{d}{0.15 \mathrm{~nm}}\right)^{2}$.

Finally, the equality of number of reducing centers in copolymer molecules covered the particle, and the amount of $\mathrm{Ag}$ atoms in the particle

$i \times \pi\left(\frac{d}{0.15 \mathrm{~nm}}\right)^{2}=4 \frac{\pi}{6}\left(\frac{d}{0.42 \mathrm{~nm}}\right)^{3}$

gives us the expression of the mode diameter depending on $\alpha$

$$
\begin{aligned}
d_{\text {mode }}(\alpha) & =\frac{6}{4} \times \frac{(0.42 \mathrm{~nm})^{3}}{(0.15 \mathrm{~nm})^{2}} \times(4-3 \alpha) \\
& \approx 5 \times(4-3 \alpha)[\mathrm{nm}] .
\end{aligned}
$$

The calculated dependence and superimposed experimental points are shown in Fig. 4 together with theoretical curves assuming that MAG possesses 1, 2, 3 , or 4 reducing sites. We see that the best agreement can be for the amount of reducing sites between 3 and 4 . 


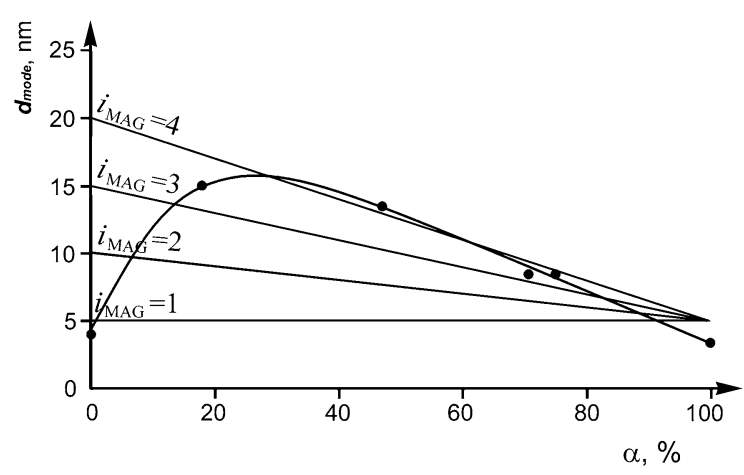

Fig. 4 Experimental points and theoretical dependence of particle mode diameter on DMAEMA mole fraction with different amounts of reducing sites in MAG monomer

The driving idea of the particle formation considers that reduced silver atoms stay close to their reducing centers, and that when a particle formation is completed, the polymer chains used for silver reduction form a tight shell around it preventing its further growth. Eventually, the balance between the polymer shell surface to give the right number of reducing centers and the volume containing the same amount of atoms defines the particle size, and diffusion in or out of this volume accounts for size distribution.
The corresponding sequence of ion reduction and particle formation is presented in Fig. 5 for the MAGDMAEMA copolymer. About 44 MAG-DMAEMA polymer molecules have to participate in reduction of silver ions in order to create $\mathrm{Ag}$ particle with the characteristic mode diameter of $8.4 \mathrm{~nm}$ (Table 3). Polymeric molecules and silver ions in the solution start their interaction (Fig. 5a) through nitrogen and oxygen reducing sites. One reducing site reduces one silver ion (Fig. 5b). Clusters of $\mathrm{Ag}^{0}$ form in the vicinity of copolymer chains (Fig. 5c) due to random collisions with atoms. The $\mathrm{Ag}$ particle grows by accretion of more atoms or clusters (Fig. 5d). At the end of the reducing/accretion process, the modified polymer chains entirely cover the particle forming tight monolayer and shield it from further accretion of $\mathrm{Ag}$ atoms or clusters (Fig. 5e). For the simplicity of the graphical model, a regular packing of polymer chains around the Ag particle is considered. However, some more irregular stacking of the chains may not be excluded from this simple model.

This model is in a good agreement with the experimental results for $\alpha$ in the range of $15-100 \%$. However, the model possesses some limitations close to $\alpha$ extreme values. Approximately, fourfold deviation

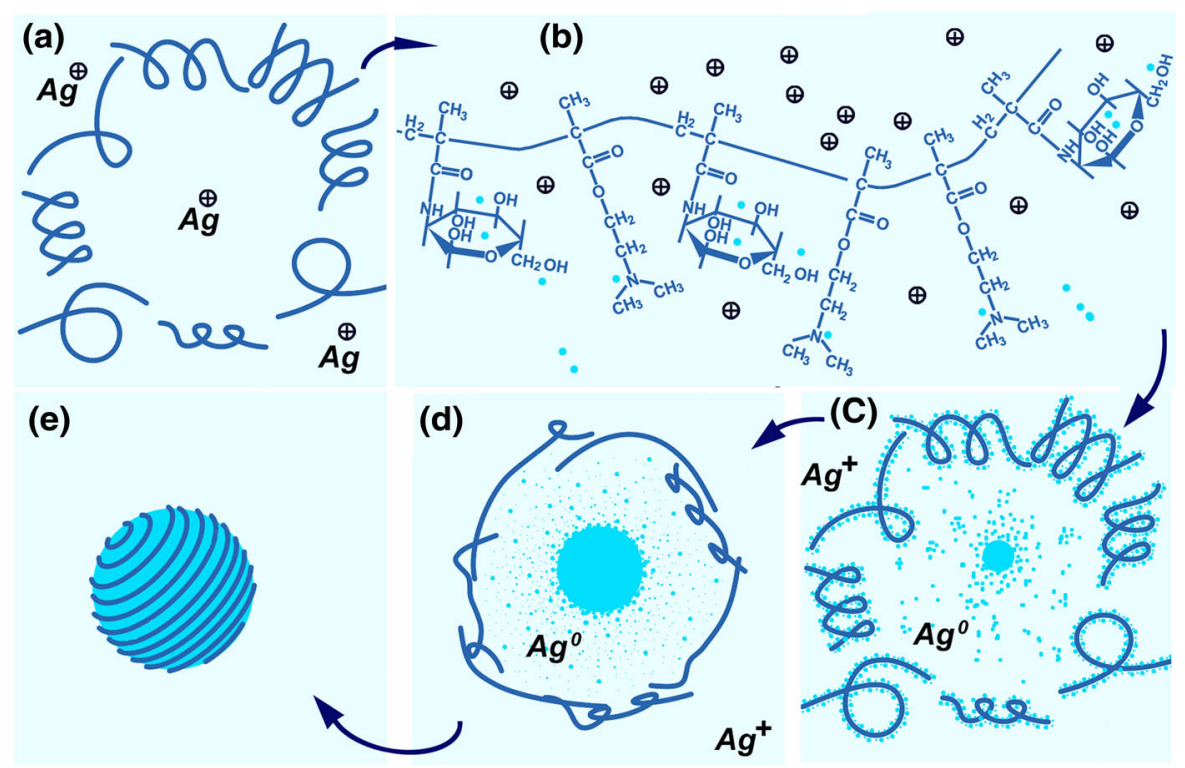

Fig. 5 Schematic presentation of the formation mechanism of an $\mathrm{Ag}$ nanoparticle with $8.4 \mathrm{~nm}$ mode diameter in the solution containing MAG-70.7 \% DMAEMA copolymer molecules of $\sim 30 \mathrm{~nm}$ in length: copolymer molecules and $\mathrm{Ag} \oplus$ ions (a), one reducing site can reduce only one $\mathrm{Ag}$ ion $\oplus$ and produce $\mathrm{Ag}$ atom blue circle (b), atoms and clusters Ag blue circle in the vicinity of copolymer chains (c), the growth of the $\mathrm{Ag}$ nanoparticle by means of accession $\mathrm{Ag}$ atoms/clusters (d), and the nanoparticle of mode diameter capped by polymer molecules modified after reaction (e). (Color figure online) 


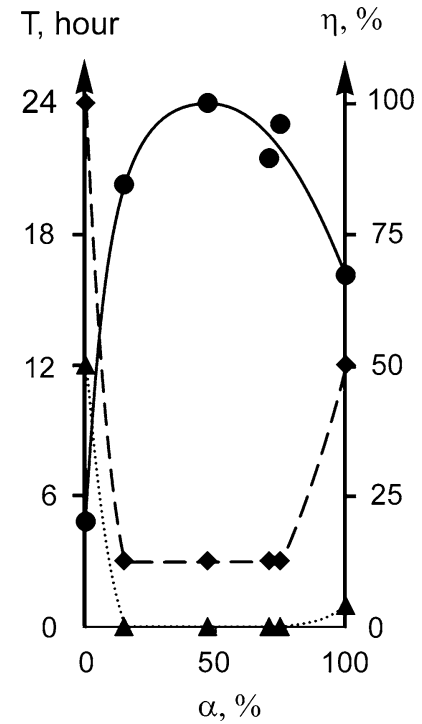

Fig. 6 Efficiency of reducing centers of homo- and copolymers (filled circle), latent periods (filled triangle), and duration $h$ of the reactions depending on the DMAEMA molar fraction $\alpha$

of the experimental $d_{\text {mode }}$ from the theoretical one (Fig. 4) was observed in pure poly[MAG] sample, and the experimental $d_{\text {mode }}$ for the pure poly[DMAEMA] sample is slightly out of the calculated values well. We remind that the reduction process of $\mathrm{Ag}$ ions in solutions of pure MAG or DMAEMA polymers has latent periods and long duration in opposite to copolymer solutions. Therefore, such deviations can be explained by different types of kinetics.

Thus it is worth to update the model and introduce depending on $\alpha$ probability $\eta(\alpha)$ of the ion reducing by polymers. We can write

$N_{\mathrm{Ag}}=\eta(\alpha) \times\langle i\rangle \times N_{\mathrm{mm}}$.

In this case, the expression for mode diameter will be written as

$d_{\text {mode }}(\alpha)=5 \times \eta(\alpha) \times(4-3 \alpha)[\mathrm{nm}]$.

The experimental results allow us to calculate probability, and the curves in Fig. 6 show variations in the reduction probability when

$\eta(\alpha)=d_{\text {mode exp }}(\alpha) /\{5 \times(4-3 \alpha)\}$.

Theoretical and experimental results agreed well. We see from the graphics in Fig. 6 that efficiency to reduce $\mathrm{Ag}$ ions reaches $100 \%$ for copolymer with $\alpha \approx 50 \%$ and lowers at $\alpha \rightarrow 0$ or $\alpha \rightarrow 100 \%$ which corresponds to pure homopolymers MAG and
DMAEMA as well correlates with the higher reaction rate for copolymers. Therefore, the $\eta(\alpha)$ dependence can be a representative of numerical characteristics for optimization of the reduction process.

One can wonder if the reducing efficiency and formation of Ag particles depends only on the quantity of MAG and DMAEMA molecules or if the grouping of these polymers in copolymer chains has a specific effect, therefore, we compared the size/volume distribution of particles prepared from solutions of MAGDMAEMA copolymers to physical mixtures of the corresponding amount of homopolymers. The sizes of particles and volume fractions of $\mathrm{Ag}$ particles formed in the solutions of $50 \%$ DMAEMA-50\% MAG and $20 \%$ DMAEMA-80\% MAG are summarized in Table SM-2 (Supplementary materials). The corresponding STEM images and the size/volume histograms are given in Figures SM-2-SM-5 in (Supplementary Materials).

A comparison was performed for solutions with pure DMAEMA polymers, pure MAG polymers, physical mixtures of $50 \%$ DMAEMA: $50 \%$ MAG and $20 \%$ DMAEMA: $80 \%$ MAG homopolymers, and the copolymers $47 \%$ DMAEMA: $53 \%$ MAG and $82 \%$ MAG: $18 \%$ DMAEMA (Supplementary Materials: Figure SM-6 and Figure SM-7). We observed that the mode diameters of $\mathrm{Ag}$ particles in both mixtures do not exceed $10 \mathrm{~nm}(4.0 \mathrm{~nm}$ for $50 \%$ poly[DMAEMA] $-50 \%$ poly[MAG] mixture and $8.0 \mathrm{~nm}$ for $20 \%$ poly[DMAEMA] $-80 \%$ poly[MAG] mixture), while the increase of molar fraction of MAG from 25 to $85 \%$ in copolymers results in the gradual increase of mode diameters up to $15 \mathrm{~nm}$. All size distributions of AgNPs formed in the mixtures and MAG homopolymer exhibit similar coarse particle tails which amount to $95 \%$ of their total volume fraction. However, the latent period characteristic for the mixture is the same (about $1 \mathrm{~h}$ ) as for the pure DMAEMA polymer solution. Therefore, we can consider that homopolymers being in the mixture reduce the silver ions and participate in the formation of particles rather independently that suggests the importance of the structure and configuration of polymers on the reducing mechanism.

\section{Bimodal size distribution}

We have seen that bimodal size distributions may exist in the MAG-18\% DMAEMA sample $(\alpha=18 \%)$ 
with maxima at 5 and $15 \mathrm{~nm}$. Bimodality indicates that several mechanisms of particle formation should be considered, for instance, multiple sources of particles or variable growth mechanisms in the system. The following explanation is suggested for the system investigated here. Particles smaller than $\sim 5 \mathrm{~nm}$ in diameter can be formed due to random collisions of metallic clusters and thus create some kind of background. When $\alpha$ is relatively large, the position of maxima is in the range of small diameters, and therefore this background is hidden. While $\alpha$ is decreasing to $18 \%$, the main maximum is located in the range of large diameters (about $15 \mathrm{~nm}$ ) allowing the background of smallest particles to become perceptible which leads to the formation of bimodal size distribution.

\section{Estimation of copolymers figure of merit for creating} small $(<10 \mathrm{~nm})$ particles

Assuming that the catalytic or bactericide activity depends only on the area of active material, in particular ignoring that the radius of curvature of the particle surface may bring extra activity, the relevant parameter becomes the particle surface $s=\pi d^{2}$. The figure of merit $F$ of the sample is its silver specific area $\left(\mathrm{m}^{2} / \mathrm{g}\right)$-i.e., the cumulative surface $S(\infty)$ of the $n(d)$ particles of diameter $d$ over the whole size distribution from $d=0$ up to the largest $D_{\infty}(d \rightarrow \infty)$-compared to the total mass $M$ of silver (density $\rho$ ) in that sample:

$F(\infty)=S(\infty) / M(\infty)$

with

$S(\infty)=\sum_{d=0}^{\infty} n(d) \times \pi d^{2}$

and

$M(\infty)=\rho \sum_{d=0}^{\infty} n(d) \times \frac{\pi}{6} d^{3}$.

The global figures of merit compare the total area of all particles to their total volume and measure the efficiency of the silver investment assuming that all silver surfaces are bioactive. Considering that only particles smaller than a critical diameter may be active, it is worth to use an effective figure of merit $F(D)=S(D) / M(\infty)$ that compares the total area

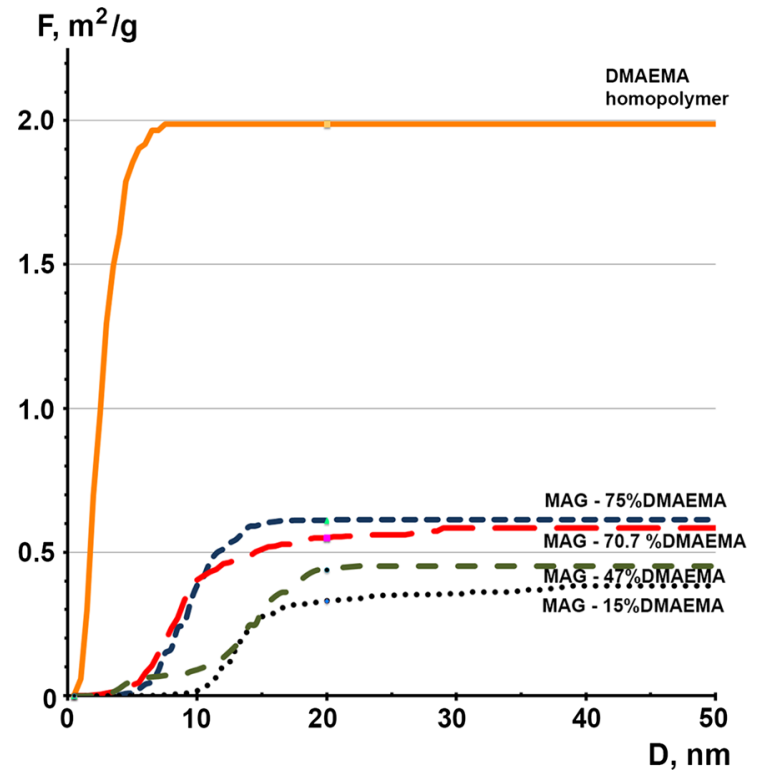

Fig. 7 Figures of merit, $\mathrm{m}^{2} / \mathrm{g}$, for the DMAEMA homopolymer and the 4 copolymers MAG-DMAEMA

$S(D)$ of the particles smaller than this critical diameter to the mass $M(\infty)$ of all particles in the sample (Fig. 7). The most striking difference between samples is the high figure of merit of DMAEMA homopolymer, about 5 times higher than for copolymers, which is still retained down to some $7 \mathrm{~nm}$ due to the small particle diameters together with the absence of a tail on the larger size side. This ratio exceeds even 20 times for the effective figure of merit if one considers that only particles smaller than $D=10 \mathrm{~nm}$ are active.

\section{Conclusions}

Ag nanoparticles were synthesized and stabilized using hydrophilic polymethylmethacrilates (DMAEMA) and polysaccharide (MAG) homopolymers, and MAG-DMAEMA copolymers with different molar fractions of DMAEMA at room temperature, atmospheric pressure, and natural light. Ag NPs in all systems were well-dispersed single crystal or sometimes twined (multiple-twinned) crystals.

The formation of Ag NPs in pure DMAEMA homopolymer started within $1 \mathrm{~h}$ and proceeded during $12 \mathrm{~h}$. It leads to the narrowest size distribution, the smallest mode diameter of $3.4 \mathrm{~nm}$ with the narrowest 
standard deviation of $0.89 \mathrm{~nm}$, and a small maximum particle size $<10 \mathrm{~nm}$. In comparison, particles formed in pure MAG homopolymer have a larger mode diameter $4.0 \mathrm{~nm}$ with $5.81 \mathrm{~nm}$ standard deviation and the large particle tail extends up to $50 \mathrm{~nm}$. It corresponds also to the largest volume fraction of big particles, and the reaction has the longest latent period and duration among all samples investigated in this work.

Mixtures of MAG and DMAEMA homopolymers start reducing silver ions after $1 \mathrm{~h}$ latent period like does the DMAEMA homopolymer but their large crystal tail amounts some $95 \%$ of the volume fraction like in MAG homopolymer. The mode diameters of Ag particles formed in the MAG/DMAEMA homopolymers mixtures are 4.0 and $8.0 \mathrm{~nm}$ for 50:50 \% and 20:80 \%, respectively.

The synthesis of Ag NPs using copolymers MAGDMAEMA with different molar fractions of DMAEMA 75.0, 70.7, 47.0, and $15.0 \%$ started after solutions mixing without a latent period and was already completed after $3 \mathrm{~h}$. Compared to the DMAEMA homopolymer, copolymers MAGDMAEMA promote the formation of larger $\mathrm{Ag}$ particles with distinct size distribution tails extending to diameters where Ag NPs are only weakly contributing to activity. The synthesis of Ag NPs in solutions of MAG-DMAEMA copolymer with $18 \%$ mole fraction of DMAEMA produces a bimodal size distribution with two peaks of diameter maxima at 5 and $15-16 \mathrm{~nm}$.

A mechanism of the formation of nanoparticles using MAG-DMAEMA polymers is proposed. One observes that the ratio of the number of reducing MAG/DMAEMA centers in a copolymer chain has a major influence on the particle size and size distribution. It can be interpreted as a fast Ag cluster formation and particle growth by subsequent atom or cluster accretion in the vicinity of the reducing chains that rapidly caps the particle and prevents further growth. Long-distance diffusion of bare particles or incompletely capped ones may lead to further accretion of larger and irregularly twinned particles. Mixtures of the same homopolymers have a mode diameter slightly larger than those of both constituents and exhibit always a tail toward the largest sizes.

A figure of merit $F=S / M$ was defined as the ratio of the total particle surface $\mathrm{S}$ to their total mass M. It represents the particle activity_assuming that it is a property of surface - to the investment cost of silver. In that sense, the DMAEMA homopolymer is the best with $2 \mathrm{~m}^{2} / \mathrm{g}$, while the figure of merit is only $0.31 \mathrm{~m}^{2} / \mathrm{g}$ for the MAG homopolymer and $0.61 \mathrm{~m}^{2} / \mathrm{g}$ for the best copolymer considered $25 \% \mathrm{MAG}: 75 \%$ DMAEMA. But if one considers that the particle curvature or dispersion is also important and that only particles smaller than a critical diameter $\mathrm{D}$ participate to activity, one define an effective figure of merit $F(D)=\mathrm{S}(\mathrm{D}) / \mathrm{M}$, where $\mathrm{S}(\mathrm{D})$ is the total surface of particles of diameter $\mathrm{D}$ or smaller. For instance, assuming that $D=10 \mathrm{~nm}$, the DMAEMA would have the best figure of merit still with $2 \mathrm{~m}^{2} / \mathrm{g}$, while it falls to $0.09 \mathrm{~m}^{2} / \mathrm{g}$ for MAG and $0.39 \mathrm{~m}^{2} / \mathrm{g}$ for $25 \%$ MAG:75 \%DMAEMA.

Acknowledgments The authors are deeply indebted to Prof. Cécile Hebert for kindly providing access to the microscopes and interpretation software of the Centre Interdisciplinaire de Microscopie Electronique of EPFL and the academician Prof. E. F. Panarin for the fruitful discussions.

\section{References}

Andreeva LN, Bezrukova MA, Bushin SV, Nekrasova TN, Imanbaev RT, Pautov VD, Zolotova YuI, Nazarova OV, Panarin EF (2014) Conformational and dynamic characteristics of copolymers of $N, N$-dimethylaminoethyl methacrylate and 2-deoxy-2-methacrylamido-D-glucose. Polym Sci A 56:405-413

Chevirona P, Gouanvéa F, Espuchea E (2014) Green synthesis of colloid silver nanoparticles and resulting biodegradable starch/silver nanocomposites. Carbohydr Polym 108:291-298

Darroudi M, Ahmad MB, Abdullah AH, Ibrahim NA, Shameli K (2010) Effect of accelerator in green synthesis of silver nanoparticles. Int J Mol Sci 11:3898-3905

Donati I, Travan A, Pelillo C, Scarpa T, Coslovi A, Bonifacio A, Sergo V, Paoletti S (2009) Polyol synthesis of silver nanoparticles: mechanism of reduction by alditol bearing polysaccharides. Biomacromolecules 10:210-213

Guinier A (1994) X-ray diffraction in crystals, imperfect crystals, and amorphous bodies. Dover Publ. Inc., New York (Chapter 5 Diffraction lines for a crystalline powder) pp 130-137

Khan Z, Al-ThabaitiS A, Al-Nowaiser FM, Obaid AY, AlYoubi AO, Malik MA (2012) Kinetics of silver nanoparticle growth in aqueous polymer solutions. Arab J Chem 5:453-459

Klomp GF, Ronel SH, Hashiguchi H, D'Andrea M, Dobelle WH (1979) Hydrogels for encapsulation of pancreatic islet cells. Trans Am Soc Artif Intern Organs 25:74-76

Korzhikov VA, Diederichs S, Nazarova OV, Vlakh EG, Kasper C, Panarin EF, Tennikova TB (2008) Water-soluble 
aldehyde-bearing polymers of 2-deoxy-2-methacrylamidoD-glucose for bone tissue engineering. J Appl Polym Sci 108:2386-2397

Li C, Yamauchi Y (2013) Facile solution synthesis of Ag@Pt core-shell nanoparticles with dendritic Pt shells. Phys Chem Chem Phys 15:3490-3496

Medina-Ramireza I, Bashirb S, Luoc Z, Liuc JL (2009) Green synthesis and characterization of polymer-stabilized silver nanoparticles. Colloids Surf B 73:185-191

Nazarova OV, Leontyeva EA, Nekrasova TN, Dobrodumov AV, Zolotova YI, Slita AV, Sushchenko EN, Malakhova II, Zelenko NN, Panarin EF (2009) Copolymers of 2-deoxy-2-methacrylamido-D-glucose with aminoacrylates and allylamine hydrochloride. J Carbohydr Chem 29:39-52

Nekrasova TN, Zolotova YI, Nazarova OV, Levit ML, Suvorova EI, Sirotkin AK, Baklagina YG, Didenko EV, Pautov VD, Panarin EF (2013) Silver nanocomposites based on (Co)polymers of 2-deoxy-2-methacrylamido-D-glucose, $\mathrm{N}$ vinylamides, and aminoacrylates. Dokl Chem 446:212-214

Rao S, Houska CR (1986) X-ray particle size broadening. Acta Cryst A42:6-13

Sharma VK, Yngard RA, Lin Y (2009) Silver nanoparticles: green synthesis and their antimicrobial activities. Adv Colloid Interface Sci 145:83-96

Sun H, Gao Z, Yang L, Gao L, Lv X (2010) Synthesis and characterization of novel four-arm star PDMAEMA-stabilized colloidal silver nanoparticles. Colloid Polym Sci 288:1713-1722

Sun H, Gao Z, Gao L, Hou K (2011) Star-PDMAEMA- $\beta$-CDstabilized colloidal gold nanoparticles: synthesis, characterization and $\mathrm{pH}$-controlled assembly. J Macromol Sci Part A 48:291-298

Tangeysh B, Fryd M, Iliesb MA, Wayland BB (2012) Palladium metal nanoparticle size control through ion paired structures of [PdCl4]2- with protonated PDMAEMA. Chem Commun 48:8955-8957

Vassilyev YuB, Khazova OA, Nikolaeva NN (1985a) Kinetics and mechanism of glucose electrooxidation on different electrode-catalysts part I. Adsorption and oxidation on platinum. J Electroanal Chem 196:105-125
Vassilyev YuB, Khazova OA, Nikolaeva NN (1985b) Kinetics and mechanism of glucose electrooxidation on different electrode-catalysts Part II. Effect of the nature of the electrode and the electrooxidation mechanism. J Electroanal Chem 196:127-144

Vigneshwaran N, Nachane RP, Balasubramanya RH, Varadarajan PV (2006) A novel one-pot 'green' synthesis of stable silver nanoparticles using soluble starch. Carbohydr Res 341:2012-2018

Voronov A, Kohut A, Vasylyev S, Peukert W (2008) Mechanism of silver ion reduction in concentrated solutions of amphiphilic invertible polyesters in nonpolar solvent at room temperature. Langmuir 24:12587-12594

Wang S, Tseng WJ (2009) Aggregate structure and crystallite size of platinum nanoparticles synthesized by ethanol reduction. J Nanopart Res 11:947-953

Wang L, Yamauchi Y (2010) Controlled aqueous solution synthesis of platinum-palladium alloy nanodendrites with various compositions using amphiphilic triblock copolymers. Chem An Asian J 5:2493-2498

Wang H, QiaoX Chen J, Wang X, Ding S (2005) Mechanisms of PVP in the preparation of silver nanoparticles. Mater Chem Phys 94:449-453

Wang L, Imura M, Yamauchi Y (2012) Tailored design of architecturally controlled Pt nanoparticles with huge surface areas toward superior unsupported Pt electrocatalysts. ACS Appl Mater Interfaces 4:2865-2869

Wu C, Mosher BP, Lyons K, Zeng T (2010) Reducing ability and mechanism for polyvinylpyrrolidone (PVP) in silver nanoparticles synthesis. J Nanosci Nanotechnol 10:2342-2347

Yasuda H (1975) Ultrathin coating by plasma polymerization applied to corneal contact lens. J Biomed Mater Res 9:629-643

Zhou L, Yuan J, Yuan W, Sui X, Wu S, Li Z, Shen D (2009) Synthesis, characterization, and controllable drug release of pH-sensitive hybrid magnetic nanoparticles. J Magnet Magnetic Mater 321:2799-2804 